\title{
Avaliação de fluxos de calor e evapotranspiração pelo modelo SEBAL com uso de dados do sensor ASTER
}

\author{
Thiago Veloso dos Santos ${ }^{(1)}$, Denise Cybis Fontana( ${ }^{(2)}$ e Rita Cássia Marques Alves ${ }^{(1)}$
}

\begin{abstract}
(1)Universidade Federal do Rio Grande do Sul (UFRGS), Centro Estadual de Pesquisas em Sensoriamento e Meteorologia, Avenida Bento Gonçalves no9.500, Caixa Postal 15.044, CEP 91501-970 Porto Alegre, RS. E-mail: thiagoveloso@gmail.com, ritacma@terra.com.br (2) UFRGS, Faculdade de Agronomia, Departamento de Plantas Forrageiras e Agrometeorologia, Avenida Bento Gonçalves no 7.712, Caixa Postal 15.100, CEP 91540-000 Porto Alegre, RS. E-mail: dfontana@ufrgs.br
\end{abstract}

Resumo - O objetivo deste trabalho foi avaliar a eficiência da aplicação do modelo SEBAL em estimar os fluxos de energia em superfície e a evapotranspiração diária, numa extensa área de cultivo de arroz irrigado, no município de Paraíso do Sul, RS, tendo como parâmetros dados do sensor ASTER. As variáveis estudadas constituem importantes parâmetros do tempo e do clima em estudos agrometeorológicos e de racionalização no uso da água. As metodologias convencionais de estimativa desses parâmetros são pontuais e geralmente apresentam incertezas, que aumentam quando o interesse é o comportamento espacial desses parâmetros. Aplicou-se o algoritmo "Surface Energy Balance Algorithm for Land" (SEBAL), em uma imagem do sensor "Advanced Spaceborne Thermal Emission and Reflection Radiometer" (ASTER). As estimativas obtidas foram comparadas com medições em campo, realizadas por uma estação micrometeorológica localizada no interior da área de estudo. As estimativas mais precisas foram as de fluxo de calor sensível e de evapotranspiração diária, e a estimativa que apresentou maior erro foi a do fluxo de calor no solo. A metodologia empregada foi capaz de reproduzir os fluxos de energia em superfície de maneira satisfatória para estudos agrometeorológicos e de rendimento de culturas.

Termos para indexação: arroz, calor latente, calor sensível, sensoriamento remoto, uso da água, resolução espacial.

\section{Evaluation of heat fluxes and evapotranspiration using SEBAL model with data from ASTER sensor}

\begin{abstract}
The objective of this study was to evaluate the efficiency of SEBAL model in estimating soil surface energy fluxes and daily evapotranspiration for a large area of irrigated rice farms, near the municipality of Paraíso do Sul, RS, Brazil, using data from ASTER sensor. The evaluated variables are important weather and climatic parameters for agrometeorological studies and rationalization of water use. The conventional methodologies for estimating these parameters generally present uncertainties, which increase when concern is in the spatial behavior of such parameters. The Surface Energy Balance Algorithm for Land (SEBAL) was applied in an Advanced Spaceborne Thermal Emission and Reflection Radiometer (ASTER) scene and the estimates were compared to micrometeorological data retrieved from a station located in the studied area. The most accurate modeled parameter estimatives were sensitive heat and evapotranspiration, and the one which presented the highest error was soil heat flux. The adopted methodology was able to reproduce surface energy fluxes for agrometeorological and crop yield studies.
\end{abstract}

Index terms: rice, latent heat, sensitive heat, remote sensing, water use, spatial resolution.

\section{Introdução}

A evapotranspiração é um dos mais importantes fatores mediadores do clima e do tempo, tanto em escala global quanto local, consistindo da ligação entre energia, clima e hidrologia. Estima-se que, por meio da evapotranspiração global, aproximadamente
$62 \%$ do volume precipitado sobre os continentes retorne à atmosfera (Dingman, 2002), fato que lhe confere o status de agente regulador fundamental das disponibilidades hídricas, superficiais e subterrâneas.

A quantificação do processo de evapotranspiração ocorre por meio de equipamentos, como lisímetros ou torres meteorológicas, usando correlações de vórtices

Pesq. agropec. bras., Brasília, v.45, n.5, p.488-496, maio 2010 
turbulentos, ou ainda por meio do balanço hídrico no solo e equações como a de Penman-Monteith (Allen et al., 1998). Porém, esses métodos podem ser considerados limitados, porque estimam valores pontuais de evapotranspiração para um local específico e não para uma escala mais ampla.

Considerando ainda o custo de instalação de complexos equipamentos de medição e a escassez de recursos humanos e econômicos, novas metodologias foram desenvolvidas para rápida obtenção de dados hidrológicos, como o uso do sensoriamento remoto. Com o objetivo de simplificar o processo de obtenção de variáveis hidrológicas para grandes áreas, alguns algoritmos têm sido desenvolvidos nos últimos anos, dentre os quais se destaca o SEBAL - "Surface Energy Balance Algorithm for Land" (Bastiaanssen, 1995; Bastiaanssen et al., 2005).

Esse algoritmo foi desenvolvido com o propósito de determinar a evapotranspiração, por meio do balanço completo da radiação e da energia incidente sobre a superfície terrestre, utilizando dados de sensoriamento remoto e alguns dados complementares de superfície, como velocidade do vento e temperatura do ar. $\mathrm{O}$ algoritmo SEBAL tem sido muito utilizado por autores brasileiros (Silva \& Bezerra, 2006; Folhes, 2007; Mendonça, 2007; Bezerra et al., 2008; Nicácio, 2008), para estimar valores de evapotranspiração consistentes, quando comparados com os medidos em campo, com variações entre 1 e $5 \%$ no resultado final. Outros pesquisadores, como Jacob et al. (2002), Hemakumara et al. (2003) e French et al. (2005), apresentam resultados com variações superiores a $20 \%$. Por também ser capaz de inferir a umidade disponível no solo, o modelo é frequentemente empregado em estudos de mapeamento de demanda hídrica de culturas e eficiência de irrigação (Zwart \& Bastiaanssen, 2007).

Na literatura, apenas Giacomoni (2005) mapeou os fluxos de calor em superfície por meio de imagens orbitais, no Rio Grande do Sul, utilizando o sensor AVHRR/NOAA, com resolução espacial média, de 250 a $1.000 \mathrm{~m}$. Portanto, é desejável que se teste a potencialidade de sensores dotados de resolução espacial mais alta. As imagens do sensor ASTER ("Advanced Spaceborn Thermal Emission and Reflection Radiometer"), integradas ao modelo SEBAL, podem ser eficientes para estimar, com adequada precisão e representação espacial, os componentes relacionados às trocas de radiação e energia entre atmosfera e a superfície.

O ASTER, um dos cinco sensores radiométricos a bordo da plataforma TERRA, possui em suas bandas resolução espacial superior à dos sensores mais comumente utilizados para estudos ambientais, como o TM/Landsat 5 e o MODIS, também presente na plataforma TERRA.

O objetivo deste trabalho foi avaliar a eficiência do algoritmo SEBAL em estimar os componentes do balanço de energia (saldo de radiação, fluxo de calor no solo, fluxos de calor sensível e latente) e da evapotranspiração realizadas com imagem do sensor ASTER, em região de produção de arroz, no Rio Grande do Sul.

\section{Material e Métodos}

A área de estudo compreendeu uma região orizicultora próxima ao município de Paraíso do Sul, localizada na região central do Rio Grande do Sul e delimitada pelas coordenadas $29^{\circ} 41^{\prime} 48^{\prime \prime} \mathrm{S}$ a $29^{\circ} 48^{\prime} 34^{\prime \prime} \mathrm{S}$ e $53^{\circ} 18^{\prime} 37^{\prime \prime} W$ a $53^{\circ} 6^{\prime} 0^{\prime \prime W}$. Segundo a classificação climática de Köeppen, o clima da região é do tipo Cfa.

A cena utilizada foi adquirida gratuitamente junto ao LP-DAAC("Land Processes DistributedActiveArchive Center"), por meio de um convênio firmado entre a agência espacial americana (National Aeronautics and Space Administration, NASA) e o Centro Estadual de Pesquisas em Sensoriamento Remoto e Meteorologia, no ano de 2002. A data de tomada da imagem foi o dia 4 de março de 2004, selecionada em função de ser a única a apresentar baixa cobertura de nuvens. Cada cena do sensor ASTER possui dimensões de $60 \times 60 \mathrm{~km}$ e resolução espacial de 15, 30 e $90 \mathrm{~m}$, respectivamente para as bandas visível, infravermelho e termal (Yamaguchi et al., 1998).

Para a implementação do algoritmo SEBAL, foram necessárias algumas informações meteorológicas da superfície a fim de estimar os componentes do balanço de energia por meio do sensoriamento remoto. Os dados meteorológicos utilizados foram provenientes de um experimento de campo conduzido no âmbito do projeto CT-Hidro (Martins, 2004; Carneiro, 2007). Nesse experimento, a plataforma instrumental foi instalada em junho de 2003 e os sensores foram fixados em uma torre, a 8 metros de altura, localizada em terreno 
plano e posicionada nas coordenadas $29^{\circ} 44^{\prime} 40^{\prime \prime} \mathrm{S}$ e $53^{\circ} 8^{\prime} 10^{\prime \prime} \mathrm{W}$.

Para o cálculo dos fluxos turbulentos de energia, que foram comparados com os resultados do algoritmo SEBAL, foram empregadas medidas de alta frequência (16 Hz) do anemômetro sônico 3D, CSAT3 (Campbell Scientific Inc., Logan, Utah, USA) e flutuações turbulentas de vapor d'água e $\mathrm{CO}_{2}$, pelo analisador de gás de caminho aberto LI-6262 (LI-COR Corporate Offices, Lincoln, Nebraska, USA), instalados no topo da torre micrometeorológica.

Os passos para a execução do algoritmo estão descritos em maiores detalhes em Allen et al. (2005). O passo inicial é a calibração radiométrica das bandas da imagem, etapa crucial para que seus pixels expressem de fato a quantidade de energia liberada por unidade de área (reflectância em superfície). Essa etapa fundamentou-se na metodologia demonstrada por Chander et al. (2007), em que os números digitais da imagem são convertidos inicialmente em radiância, ou seja, a energia captada pelo sensor é transformada em reflectância aparente no topo da atmosfera, por meio de uma expressão linear aplicada em cada banda da imagem. A reflectância de superfície é obtida depois de removidos os efeitos de absorção, espalhamento e reflexão que a atmosfera exerce sobre a radiação, por meio de modelos baseados em transferência radiativa tais como o 5S/6S (Tanré et al., 1990) ou o MODTRAN (Berk et al., 2000).

Em seguida, são derivados índices de vegetação a partir da reflectância de superfície, que indicam o tipo de cobertura do solo que entrará no cômputo da emissividade da superfície. O albedo também é estimado a partir da reflectância de superfície. O saldo de radiação incorpora todas as informações de superfície obtidas (albedo, temperatura e emissividade). O fluxo de calor no solo, fluxo de calor sensível e saldo de radiação são somados para determinação do fluxo de calor latente.

Para as superfícies líquidas, ou seja, as que possuem índice de vegetação por diferença normalizada (NDVI) negativo, Allen et al. (2005) consideram o termo G como sendo metade do saldo de radiação. No Brasil, Silva \& Bezerra (2006) sugeriram a adoção do fluxo de calor no solo como uma fração de $30 \%$ do saldo de radiação, por considerarem que os reservatórios de água da área de interesse diferiam das condições propostas pelos autores americanos. Portanto, esta foi a razão adotada neste trabalho.

O Saldo ou Balanço de Radiação indica a radiação disponível em superfície, em que devem ser consideradas tanto a radiação de onda longa quanto a radiação de onda curta. Neste trabalho adotou-se a seguinte equação para o cálculo do saldo de radiação $\left(\mathrm{R}_{\mathrm{n}}\right)$ :

$$
\mathrm{R}_{\mathrm{n}}=\left(1-\alpha_{\text {sup }}\right) \mathrm{R}_{\mathrm{c}} \downarrow+\mathrm{R}_{\mathrm{L}} \downarrow-\mathrm{R}_{\mathrm{L}} \uparrow-\left(1-\varepsilon_{\mathrm{s}}\right) \mathrm{R}_{\mathrm{L}} \downarrow
$$

em que: $\alpha_{\text {sup }}$ é o albedo da superfície (adimensional), $R_{c} \downarrow$ representa a radiação de onda curta incidente ( $\left.\mathrm{W} \mathrm{m}^{-2}\right), \mathrm{R}_{\mathrm{L}} \downarrow$ é a radiação de onda longa incidente $\left(\mathrm{W} \mathrm{m}^{-2}\right), \mathrm{R}_{\mathrm{L}} \uparrow$ é a radiação de onda longa emitida pela superfície $\left(\mathrm{W} \mathrm{m}^{-2}\right)$ e $\varepsilon_{\mathrm{s}}$ é a emissividade da superfície (adimensional).

O algoritmo SEBAL computa a razão entre o fluxo de calor no solo e o saldo de radiação a partir de uma equação empírica, que representa valores próximos ao meio-dia:

$\mathrm{G}=\left[\left(\mathrm{T}_{\mathrm{S}} / \alpha_{\text {sup }}\right)\left(0,0038 \alpha_{\text {sup }}+0,0074 \alpha_{\text {sup }}^{2}\right)\left(1-0,98 \mathrm{NDVI}^{4}\right)\right]$ $\mathrm{R}_{\mathrm{n}}$

em que: $T_{s}$ é a temperatura de superfície $\left({ }^{\circ} \mathrm{C}\right)$, e o NDVI é o índice de vegetação por diferença normalizada (adimensional).

A determinação do fluxo de calor sensível (H) é a etapa mais importante do modelo SEBAL. Sua estimativa é complexa e requer uma série de suposições para ser corretamente computada. $\mathrm{H}$ foi calculado por: $\mathrm{H}=\left(\rho \mathrm{C}_{\mathrm{p}} \mathrm{dT}\right) / \mathrm{r}_{\mathrm{ah}}$, em que: $\rho$ é a densidade do ar $\left(1,15 \mathrm{~kg} \mathrm{~m}^{-3}\right), \mathrm{C}_{\mathrm{p}}$ é o calor específico do ar à pressão constante de $1.004 \mathrm{~J} \mathrm{~kg}^{-1} \mathrm{~K}^{-1}$, dT é a diferença de temperatura $(\mathrm{K})$ entre dois níveis, e $\mathrm{r}_{\mathrm{ah}}$ é a resistência aerodinâmica ao transporte de calor $\left(\mathrm{s} \mathrm{m}^{-1}\right)$. O processo para determinação do fluxo de calor sensível, incluindo a correção da estabilidade atmosférica (Bezerra et al., 2008; Santos, 2009), consiste em um processo iterativo que envolve a resistência aerodinâmica da superfície, estimada a partir de um registro de velocidade de vento, e a localização na imagem de pontos chamados de pixels-âncora.

Esses pontos refletem duas condições extremas de fluxo de calor: o pixel frio deve se localizar em uma área irrigada de cultivo na qual se assumirá que o fluxo de calor sensível é nulo, e o de calor latente é máximo. 
Em contraste, o pixel quente deve ser selecionado sobre uma área de solo exposto, onde não há umidade disponível para a ocorrência de evapotranspiração. Nesse caso, o fluxo de calor latente pode ser desprezado e o fluxo de calor sensível é máximo.

No modelo SEBAL, o fluxo de calor latente (LE, $\mathrm{W} \mathrm{m}^{-2}$ ) foi estimado como resíduo da equação do balanço energético, levando-se em consideração a diferença entre $R_{n}\left(W^{-2}\right), G\left(W m^{-2}\right)$ e $H\left(W m^{-2}\right)$, conforme a equação: $\mathrm{LE}=\mathrm{R}_{\mathrm{n}}-\mathrm{G}-\mathrm{H}$.

O fluxo de calor latente foi posteriormente convertido em evapotranspiração, por meio da aplicação de sua relação com o calor latente de vaporização da água, definido como a energia necessária, em Joules, para evaporar uma massa unitária de água $-\lambda$, conforme a expressão: $\mathrm{ET}_{\mathrm{h}}=3600(\mathrm{LE} / \lambda)$, em que $\lambda$ é expresso em $\mathrm{J} \mathrm{kg}^{-1}$ e $E T_{\mathrm{h}}$ representa a evapotranspiração horária, estimada para o horário de passagem do satélite, expressa em $\mathrm{mm}$. Esse valor de $\mathrm{ET}_{\mathrm{h}}$ foi, em seguida, extrapolado para valores diários por meio da equação, proposta por Bastiaanssen (1995), que relaciona o fluxo de calor latente com a integração diária dos valores de saldo de radiação.

Para a determinação do fluxo calor sensível, os pixels frio e quente foram eleitos, respectivamente, sobre uma porção da imagem que continha uma lavoura irrigada de arroz e sobre uma região de campo exposto. A estabilização dos valores de resistência
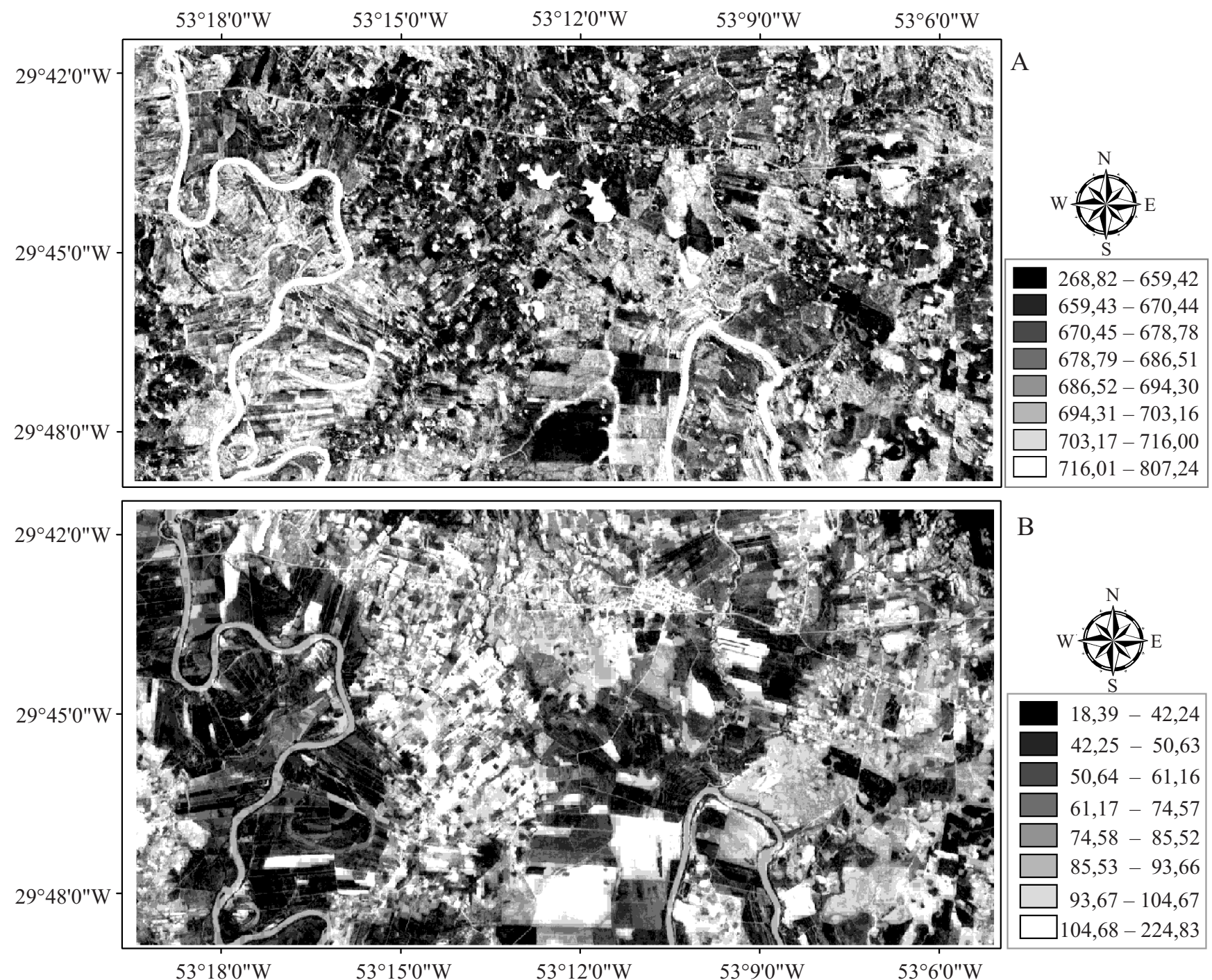

Figura 1. Distribuição espacial do saldo de radiação (A) e fluxo de calor no solo (B), no dia 4 de março de 2004. Os valores da escala de cinza estão expressos em $\mathrm{W} \mathrm{m}^{-2}$. 
aerodinâmica e diferença de temperatura foi alcançada após seis repetições no processo iterativo que envolve os pixels-âncora.

\section{Resultados e Discussão}

A Figura 1 A apresenta a imagem dos valores instantâneos do saldo de radiação à superfície $\left(R_{n}\right)$, no momento da passagem do satélite. As áreas mais escuras representam as áreas da superfície com valores de $R_{n}$ inferiores a $678 \mathrm{~W} \mathrm{~m}^{-2}$. Essas áreas foram caracterizadas como áreas de solo exposto ou com vegetação nativa pouco densa, e coincidem, dessa forma, com os valores encontrados por Bezerra (2006) em áreas com características similares, em estudo de quantificação dos componentes do balanço de energia, com uso de imagens Landsat 5-TM e do algoritmo SEBAL, em áreas irrigadas do Projeto Nilo Coelho e adjacências, no município de Petrolina, PE.

As tonalidades intermediárias de cinza $-R_{n}$ de 678 a $703 \mathrm{~W} \mathrm{~m}^{-2}$ - representam as áreas com cobertura vegetativa de densidade intermediária, que envolvem vegetação nativa e áreas utilizadas na prática de agricultura irrigada, em início do cultivo. As áreas com as tonalidades mais claras de cinza representam valores de $\mathrm{R}_{\mathrm{n}}$ acima de $703 \mathrm{~W} \mathrm{~m}^{-2}$. Observa-se que esses valores concentram-se na vegetação de maior densidade, que correspondem às culturas de arroz e soja, e nos espelhos d'água dos açudes localizados na parte superior das imagens. Paiva (2005) e Bezerra (2006) encontraram valores semelhantes, respectivamente, para o lago de Sobradinho, na Bahia, e para uma fazenda de soja em Dourados, MS.

Leivas (2008) encontrou valores de $585 \mathrm{~W} \mathrm{~m}^{-2}$ para regiões de solo exposto, $662 \mathrm{~W} \mathrm{~m}^{-2}$ para corpos d'água e em torno de $600 \mathrm{~W} \mathrm{~m}^{-2}$ para superfícies vegetadas. Em relação à concordância entre o valor estimado pelo algoritmo no atual trabalho $\left(687 \mathrm{~W} \mathrm{~m}^{-2}\right)$ e a medição pelo instrumento $\left(558 \mathrm{~W} \mathrm{~m}^{-2}\right)$, a diferença no saldo de radiação foi de aproximadamente $23 \%$.

Ao se observar a espacialização de G (Figura 1B), pode-se perceber valores de menor magnitude nas áreas mais úmidas (corpos hídricos e alagados), e maiores nas áreas urbanas e nas áreas desprovidas de vegetação. Destaca-se, no centro do recorte, a cidade de Paraíso do Sul, onde o fluxo de calor no solo é mais elevado, o que indica a ocorrência de ilhas de calor, fenômeno frequentemente observado em áreas urbanas
(Weng et al., 2004). As regiões de solo desprovido de cobertura na porção inferior da imagem foram as que apresentaram os índices mais elevados de fluxo de calor no solo, com valores superiores a $100 \mathrm{~W} \mathrm{~m}^{-2}$. De maneira similar, Bezerra (2006) encontrou a faixa de 115 a $190 \mathrm{~W} \mathrm{~m}^{-2}$ para áreas descobertas ou com vegetação nativa rala.

Neste trabalho, não houve similaridade entre a estimativa de $\mathrm{G}$ e a medida realizada pelo fluxímetro HFT3 (Campbell Scientific Inc., Logan, Utah, USA), que foram $68 \mathrm{~W} \mathrm{~m}^{-2}$ e $-20 \mathrm{~W} \mathrm{~m}^{-2}$, respectivamente. A mesma conclusão foi alcançada por Folhes (2007), que detectou uma diferença de $227 \%$ entre estimativa e medição de fluxo de calor no solo. Esse autor creditou a discrepância observada à instalação das placas de fluxo de calor próximo à linha de irrigação. A situação desta pesquisa é similar, já que o solo onde os fluxímetros foram instalados se encontrava alagado em boa parte do ano. $\mathrm{O}$ resultado poderia ter melhorado com uma medida de $\mathrm{G}$ mais representativa da parcela agrícola, com uso de maior número de placas de fluxo de calor e de maior espaçamento entre elas.

$\mathrm{Na}$ Figura 2A estão apresentados os valores instantâneos de H. A imagem mostra menor nitidez em virtude da incorporação da banda termal, que possui resolução espacial seis vezes menor do que as bandas visíveis do sensor, com resolução de 15 metros. Nessa imagem de $\mathrm{H}$, os valores inferiores a $72 \mathrm{~W} \mathrm{~m}^{-2}$ estão representados pelas cores mais escuras. Observa-se que estes valores concentram-se principalmente sobre as lavouras de arroz. Os pixels com valores de $\mathrm{H}$ menores que zero correspondem aos pixels contendo massa de água. Além disso, é possível perceber ainda que regiões urbanizadas, como a cidade de Paraíso do Sul, foram marcadas por valores de calor sensível entre 200 e 400 $\mathrm{W} \mathrm{m}^{-2}$, resultado semelhante ao encontrado por Nicácio (2008) para o município de Petrolina. Os valores mais elevados de calor sensível encontram-se nas regiões dotadas de menores coberturas vegetativas (NDVI inferior a 0,4$)$. Também espera-se que $\mathrm{H}$ tenha relação de proporcionalidade direta com a temperatura. No pixel que contém a torre meteorológica, o valor modelado de $\mathrm{H}$ foi de 19,3 W m ${ }^{-2}$, e apresenta alta concordância com o valor medido pela estação: $21,3 \mathrm{~W} \mathrm{~m}^{-2}$.

A variação espacial do fluxo de calor latente obtida nesta pesquisa pode ser observada na Figura 2 B. A partir da metodologia adotada, o fluxo de calor latente $(\lambda \mathrm{ET})$ foi calculado como um termo residual do balanço de 
energia, conforme sugerido por Bastiaanssen (1995). $\mathrm{Na}$ figura, os valores compreendidos entre -108 e 296 $\mathrm{W} \mathrm{m}^{-2}$ estão representados pela cor preta. A intensidade da cor é diretamente proporcional à densidade de vegetação, ou seja, áreas dotadas de vegetação nativa apresentam-se nos tons de cinza intermediários. Em contrapartida, as regiões de solo exposto, quase desnudas, apresentam os menores valores de $\lambda E T$, tendendo a tons mais escuros de cinza.

Uma outra feição notável na imagem são as áreas preenchidas pela cor branca, que cobrem a faixa entre 633 e $778 \mathrm{~W} \mathrm{~m}^{-2}$ e representam as lavouras de arroz e os corpos de água presentes na cena. Na imagem, é possível perceber que, em virtude da confusão espectral entre água e vegetação, o fluxo de calor latente nas lavouras no início do desenvolvimento assemelha-se ao observado sobre as massas de água. Em contraste, nas lavouras mais desenvolvidas, o fluxo de calor latente tende a exibir menores valores.

Em relação à exatidão do modelo SEBAL, Ma et al. (2004) relatam erro percentual de menos de $10 \%$ nas estimativas de $\lambda E T$ modeladas com auxílio das imagens $\mathrm{TM} /$ Landsat 5, quando comparadas a medidas obtidas por sensores instalados em torres micrometeorológicas. Jacob et al. (2002) e French et al. (2005), entre outros, encontraram piores concordâncias entre as medidas de $\lambda$ ET feitas pelo método das correlações turbulentas e as obtidas pela modelagem realizada com base nos dados

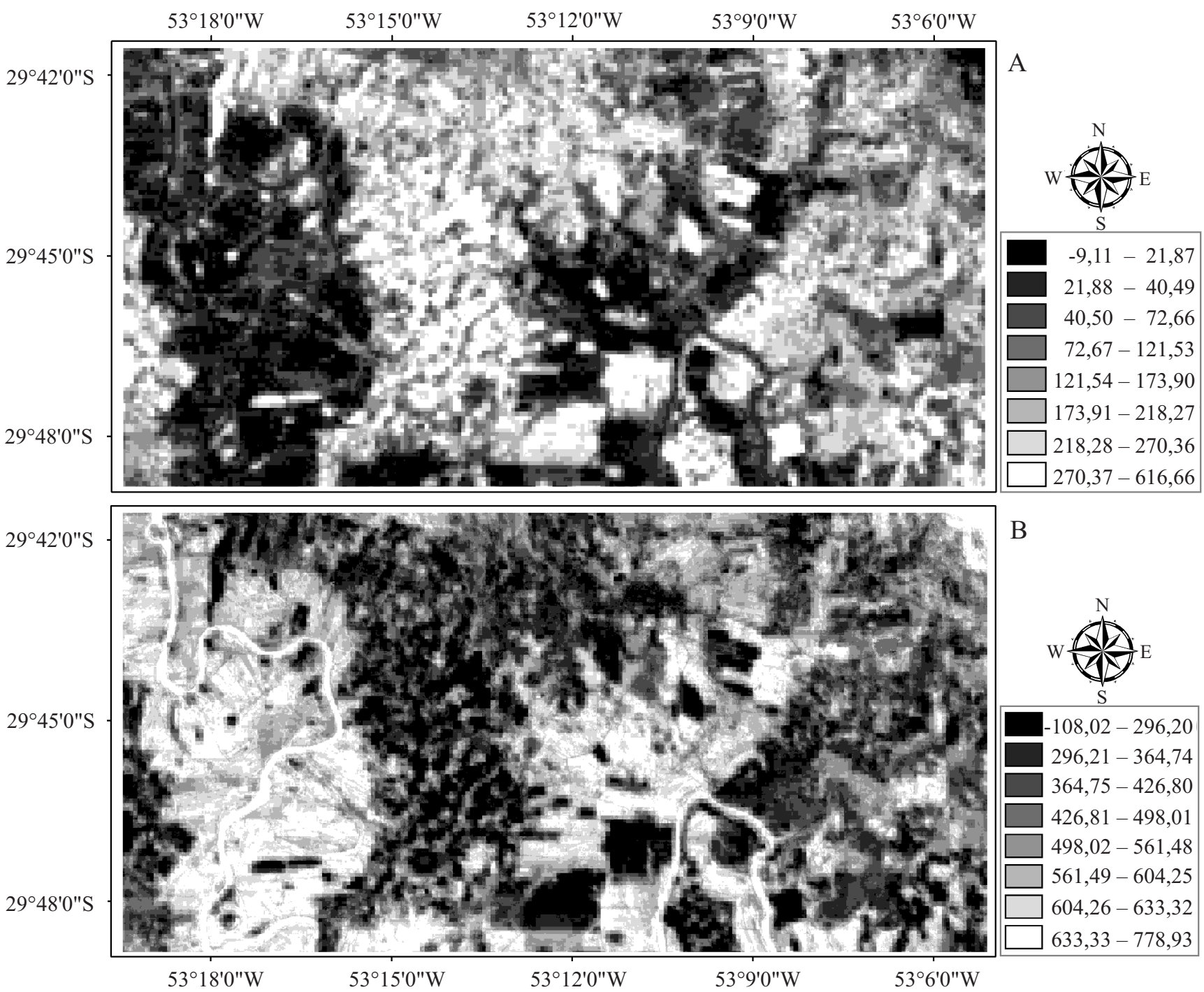

Figura 2. Distribuição espacial do fluxo de calor sensível (A) e fluxo de calor latente (B), no dia 4 de março de 2004. Os valores da escala de cinza estão expressos em $\mathrm{W} \mathrm{m}^{-2}$. 
espectrais contidos nas imagens de sensores orbitais e aerotransportados.

Por causa de uma falha no sensor de umidade, não foi possível determinar o fluxo de calor latente a partir do método das correlações turbulentas, para que se pudesse realizar a comparação com a estimativa do algoritmo SEBAL. Martins (2004) também reportou dificuldades na aquisição dos dados, no sítio de Paraíso do Sul. Em seu trabalho, somente 12 dias de dados brutos puderam ser aproveitados dos 30 dias do mês de julho de 2003. Em estudo subsequente, Martins (2008) complementa essa questão, ao afirmar que existem heterogeneidades próprias da cultura de arroz que podem adulterar o campo turbulento, heterogeneidades estas que se manifestam nos dados como grandes desvios da média, em séries temporais de grandezas turbulentas.

A evapotranspiração do arroz irrigado por inundação depende da demanda evaporativa da atmosfera e é pouco dependente da fase de desenvolvimento da cultura (Khandelwal, 1991). Assim, a taxa de evapotranspiração está relacionada com temperatura e umidade do ar, movimento do vento e intensidade e duração da luz solar e, em menor escala, com o número e tamanho das folhas. No início do ciclo da cultura, a evapotranspiração é, em sua maior parte, composta de evaporação da superfície da água, mas, à medida que a cultura cresce e sombreia a água, a evaporação decresce e aumenta a transpiração do dossel.
Segundo Tabbal et al. (2002), valores típicos de evapotranspiração de arroz nos trópicos são 4 a $5 \mathrm{~mm}$ dia $^{-1}$, na estação chuvosa, e 6 a $7 \mathrm{~mm} \mathrm{dia}^{-1}$, na estação seca. O mapeamento das taxas de evapotranspiração em Paraíso do Sul, segundo a metodologia SEBAL (Figura 3), apresenta concordância com esses valores. As lavouras de arroz, predominantemente marcadas com níveis claros de cinza, apresentaram evapotranspiração diária da ordem de 5,81 a 7,81 mm dia $^{-1}$, no dia 4 de março de 2004.

As áreas de solo exposto, marcadas com a cor preta na imagem, apresentaram os menores valores de ET, com valores inferiores a 3,96 mm $\mathrm{dia}^{-1}$. As áreas com vegetação nativa presente, destacadas em tons médios de cinza na imagem, apresentaram valores de ET entre 3,97 e 5,80 $\mathrm{mm} \mathrm{dia}^{-1}$. Nos casos em que há disponibilidade de água na superfície, a tendência preferencial de consumo de energia do $R_{n}$ é na forma de calor latente. Em estudo sobre uma região que apresentava cobertura vegetal muito heterogênea no Sri Lanka, Hemakumara et al. (2003) obtiveram valores diários de 3,0 a 4,0 mm, com uso de um cintilômetro de grande abertura. Em estudo no Novo México, na região de Las Cruces, composta por desertos e áreas de culturas irrigadas, Wang et al. (2005) aplicaram o algoritmo SEBAL adaptado a imagens ASTER e obtiveram, em áreas com cultura irrigada, valores de ET na faixa de 4,9 a 5,9 $\mathrm{mm} \mathrm{dia}^{-1}$. As massas de água representadas na Figura 3 mostram valores diários de ET entre $6,41 \mathrm{e} 7,81 \mathrm{~mm} \mathrm{dia}^{-1}$. Allen et al. (2005), com auxílio do modelo METRIC, similar

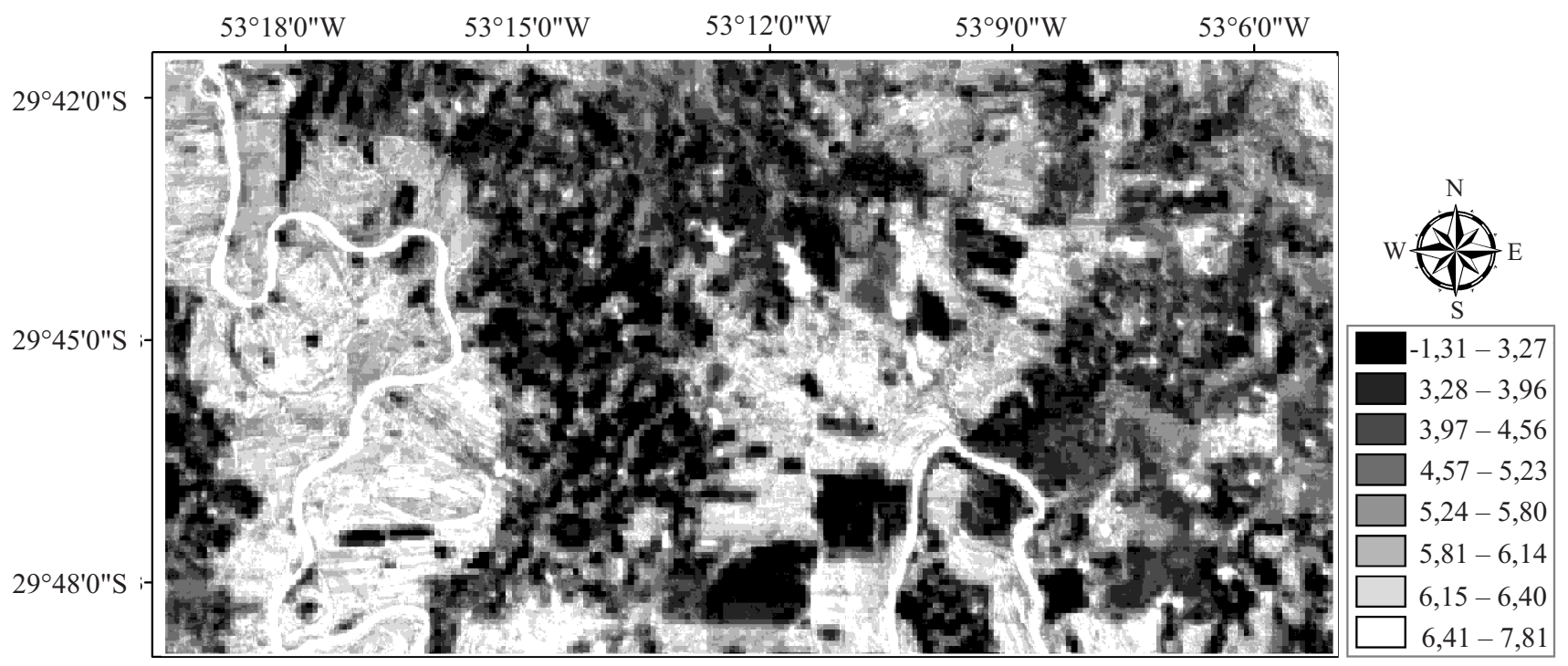

Figura 3. Distribuição espacial da evapotranspiração ( $\left.\mathrm{mm} \mathrm{dia}^{-1}\right)$, no dia 4 de março de 2004. 
ao SEBAL, nas bacias dos rios Bear, sudeste de Idaho, e Snake Plain, leste de Idaho, obtiveram valores de ET iguais a 7,17 e 7,40 $\mathrm{mm} \mathrm{dia}^{-1}$, para os dias 23/7/1989 e 25/9/1989, respectivamente.

$\mathrm{Na}$ água, a maior quantidade de energia é destinada ao processo evapotranspirativo, na forma de calor latente. O mesmo ocorre, porém com amplitude menor, sobre as culturas de arroz presentes na cena. Regiões de solo exposto, ao contrário, possuem maior parte da radiação destinada ao aquecimento do solo.

\section{Conclusões}

1. Os dados de fluxo de energia, estimados com o sensor de alta resolução espacial ASTER, reproduzem corretamente os fluxos de energia na superfície da área analisada e permitem a estimativa da variabilidade espacial da evapotranspiração.

2. O modelo SEBAL com uso de imagens do sensor ASTER é eficiente para estimar os fluxos de energia em superfície e a evapotranspiração diária em regiões de cultivo de arroz.

\section{Agradecimentos}

Ao Dr. Osvaldo Luiz Leal de Moraes e a Janaína Viário Carneiro, do Laboratório de Micrometeorologia da Universidade Federal de Santa Maria, pela concessão dos dados meteorológicos utilizados nessa pesquisa; ao Conselho Nacional de Desenvolvimento Científico e Tecnológico, pela bolsa de mestrado ao primeiro autor.

\section{Referências}

ALlEN, R.G.; PEREIRA, L.S.; RAES, D.; SMITH, M. Crop evapotranspiration: guidelines for computing crop water requirements. Rome: FAO, 1998. 300p. (FAO. Irrigation and drainage paper, 56).

ALLEN, R.G.; TASUMI, M.; MORSE, A.; TREZZA, R. A Landsat-based energy balance and evapotranspiration model in Western US water rights regulation and planning. Irrigation and Drainage Systems, v.19, p.251-268, 2005.

BASTIAANSSEN, W.G.M. Regionalization of surface flux densities and moisture indicators in composite terrain: a remote sensing approach under clear skies in Mediterranean climates. 1995. 273p. Thesis (Ph.D.) - Wageningen Agricultural University, Wageningen.

BASTIAANSSEN, W.G.M.; NOORDMAN, E.J.M.; PELGRUM, H.; DAVIDS, G. SEBAL model with remotely sensed data to improve water-resources management under actual field conditions. Journal of Irrigation and Drainage Engineering, v.131, p.85-93, 2005.

BERK, A.; ACHARYA, P.K.; BERNSTEIN, L.S.; ANDERSON, G.P.; CHETWYND, J.H.; HOKE, M.L. Reformulation of the MODTRAN band model for higher spectral resolution. Proceedings of SPIE, v.4049, p.190-198, 2000.

BEZERRA, B.G. Balanço de energia e evapotranspiração em áreas com diferentes tipos de cobertura de solo no Cariri Cearense através do algoritmo SEBAL. 2006. 127p. Dissertação (Mestrado) - Universidade Federal de Campina Grande, Campina Grande.

BEZERRA, B.G.; SILVA, B.B. da; FERREIRA, N.J. Estimativa da evapotranspiração real diária utilizando-se imagens digitais TM-Landsat 5. Revista Brasileira de Meteorologia, v.23, p.305-317, 2008.

CARNEIRO, J.V. Fluxos turbulentos de $\mathrm{CO}_{2}$ em uma área de arroz irrigado no RS. 2007. 57p. Dissertação (Mestrado) Universidade Federal de Santa Maria, Santa Maria.

CHANDER, G.; MARKHAM, B.L.; BARSI, J.A. Revised Landsat-5 thematic mapper radiometric calibration. IEEE Geoscience and Remote Sensing Letters, v.4, p.490-494, 2007.

DINGMAN, S.L. Physical hydrology. Long Grove: Prentice Hall, 2002. 600p.

FOLHES, M.T. Modelagem da evapotranspiração para a gestão hídrica de perímetros irrigados com base em sensores remotos. 2007. 189p. Tese (Doutorado) - Instituto Nacional de Pesquisas Espaciais, São José dos Campos.

FRENCH, A.N.; JACOB, F.; ANDERSON, M.C.; KUSTAS, W.P.; TIMMERMANS, W.; GIESKE, A.; SU, Z.; SU, H.; MCCABE, M.F.; LI, F.; PRUEGER, J.; BRUNSELL, N. Surface energy fluxes with the Advanced Spaceborne Thermal Emission and Reflection radiometer (ASTER) at the Iowa 2002 SMACEX site (USA). Remote Sensing of Environment, v.99, p.55-65, 2005.

GIACOMONI, M.H. Estimativa da evapotranspiração regional por meio de técnicas de sensoriamento remoto integradas a modelos de balanço de energia: aplicação no estado do Rio Grande do Sul. 193p. 2005. Dissertação (Mestrado) - Universidade Federal do Rio Grande do Sul, Porto Alegre.

HEMAKUMARA, H.M.; CHANDRAPALA, L.; MOENE, A.F. Evapotranspiration fluxes over mixed vegetation areas measured from large aperture scintillometer. Agricultural Water Management, v.58, p.109-112, 2003.

JACOB, F.; OLIOSO, A.; GU, X.; SU, Z.; SEGUIN, B. Mapping surface fluxes using airborne visible, near infrared, thermal infrared remote sensing and a spatialized surface energy balance model. Agronomie, v.22, p.669-680, 2002.

KHANDELWAL, M.K. Meteorological aspects of wet season rice cultivation in Sunderbans region, India. International Rice Research Newsletter, v.16, p.25-26, 1991.

LEIVAS, J. Uso de técnicas de sensoriamento remoto para obtenção do saldo de radiação na superfície. 2008. 107p. Tese (Doutorado) - Universidade Federal do Rio Grande do Sul, Porto Alegre. 
MA, Y.M.; MENENTI, M.; TSUKAMOTO, O.; ISHIKAWA, H.; WANG, J.M.; GAO, Q. Remote sensing parameterization of regional land surface heat fluxes over arid area in northwestern China. Journal of Arid Environments, v.57, p.257-273, 2004.

MARTINS, C.A. Características da turbulência: correlações e espectros em diferentes topografias. 2008. 164p. Tese (Doutorado) - Universidade Federal de Santa Maria, Santa Maria.

MARTINS, C.A. Comparação dos fluxos turbulentos em diferentes condições de estabilidade e topografia. 2004. 1034p. Dissertação (Mestrado) - Universidade Federal de Santa Maria, Santa Maria.

MENDONÇA, J.C. Estimação da evapotranspiração regional utilizando imagens digitais orbitais na região Norte Fluminense, RJ. 2007. 167p. Tese (Doutorado) - Universidade Estadual do Norte Fluminense Darcy Ribeiro, Campo dos Goytacazes.

NICÁCIO, R.M. Evapotranspiração real e umidade do solo usando dados de sensores orbitais e a metodologia SEBAL na bacia do rio São Francisco. 2008. 337p. Tese (Doutorado) Universidade Federal do Rio de Janeiro, Rio de Janeiro.

PAIVA, C.M. Estimativa do balanço de energia e da temperatura da superfície via satélite NOAA-AVHRR. 2005. 248p. Tese (Doutorado) - Universidade Federal do Rio de Janeiro, Rio de Janeiro.

SANTOS, T.V. Fluxos de calor na superfície e evapotranspiração diária em áreas agrícolas e de vegetação nativa na bacia do Jacuí por meio de imagens orbitais. 2009. 96p. Dissertação (Mestrado) - Universidade Federal do Rio Grande do Sul, Porto Alegre.

SILVA, B.B. da; BEZERRA, M.V.C. Determinação dos fluxos de calor sensível e latente na superfície utilizando imagens TM
- Landsat 5. Revista Brasileira de Agrometeorologia, v.14, p.174-186, 2006.

TABBAL, D.F.; BOUMAN, B.A.M.; BHUIYAN, S.I.; SIBAYAN, E.B.; SATTAR, M.A. On-farm strategies for reducing water input in irrigated rice: case studies in the Philippines. Agricultural Water Management, v.56, p.93-112, 2002.

TANRÉ, D.; DEROO, C.; DUHAUT, P.; HERMAN, M.; MORCRETTE, J.J.; PERBOS, J.; DESCHAMPS, P.Y. Description of a computer code to simulate the satellite signal in the solar spectrum: the $5 \mathrm{~S}$ code. International Journal of Remote Sensing, v.11, p.659-668, 1990.

WANG, J.; SAMMIS, T.W.; MEIER, C.A.; SIMMONS, L.J.; SAMANI, Z. A modified SEBAL model for spatially estimating Pecan consumptive water use for Las Cruces, New Mexico. In: CONFERENCE ON APPLIED CLIMATOLOGY, 15.; SYMPOSIUM ON METEOROLOGICAL OBSERVATIONS AND INSTRUMENTATION, 13., 2005, Savannah. Proceedings. Savannah: AMS Committee on Applied Climatology, 2005. 9p.

WENG, Q.H.; LU, D.S.; SCHUBRING, J. Estimation of land surface temperature-vegetation abundance relationship for urban heat island studies. Remote Sensing of Environment, v.89, p.467-483, 2004

YAMAGUCHI, Y.; KAHLE, A.B.; TSU, H.; KAWAKAMI, T.; PNIEL, M. Overview of Advanced Spaceborne Thermal emission and Reflection Radiometer (ASTER). IEEE Transactions on Geoscience and Remote Sensing, v.36, p.1062-1071, 1998.

ZWART, S.J.; BASTIAANSSEN, W.G.M. SEBAL for detecting spatial variation of water productivity and scope for improvement in eight irrigated wheat systems. Agricultural Water Management, v.89, p.287-296, 2007.

Recebido em 3 de abril de 2010 e aprovado em 18 de maio de 2010

Pesq. agropec. bras., Brasília, v.45, n.5, p.488-496, maio 2010 